\title{
Canonical cell model of cadmium-based icosahedral alloys
}

\author{
M. MIHALKOVIČ $†$ and M. WIDOM*† \\ $\dagger$ Institute of Physics, Slovak Academy of Sciences, 84511 Bratislava, Slovakia \\ tDepartment of Physics, Carnegie Mellon University, Pittsburgh, \\ Pennsylvania 15213, USA
}

(Received 14 May 2005; in final form 23 August 2005)

\begin{abstract}
Cadmium-based quasicrystals $(\mathrm{Cd}-\mathrm{Ca}$ and $\mathrm{Cd}-\mathrm{Yb})$ were the first binary alloys discovered to form thermodynamically stable quasicrystals. As binary alloys, and with a strong size difference between atomic species, they are ideal systems for structural and thermodynamic analysis. Observed quasicrystal approximants with crystallographically determined structures can be interpreted as decorations of Henley's canonical cells. We use first-principles total energy calculations to resolve details of the most favourable decorations.
\end{abstract}

\section{Introduction}

Thermodynamically stable binary icosahedral quasicrystals (iQCs) occur in the alloy systems $\mathrm{Cd}_{5.7} \mathrm{Yb}$ and $\mathrm{Cd}_{5.7} \mathrm{Ca}$ [1-3]. Since their discovery, numerous attempts have been made to model their structure. Efforts that rely on 'atomic surfaces' and higher-dimensional space [4,5] offer only an averaged picture of the structure, but the ultimate goal, namely understanding of physical properties and mechanism of stability, requires a study of structures that are energetically plausible in every detail. This task is specifically difficult for icosahedral CaCd-type structures, owing to the strong displacive symmetry breakings induced by cluster centre tetrahedron configurations, whose relative orientations may be coupled according to how the clusters are linked along twofold and threefold icosahedral directions. We use first-principles total energy calculations in combination with a tiling decoration approach, to optimize our models and to resolve details not available from the known approximants, either because of intrinsic structural disorder in the materials or because of the limited number of known structures that can be used as examples.

Assuming that the fundamental building motif of the structure is the large $\mathrm{Ca}_{12} \mathrm{Cd}_{54}$ atom cluster (see figure 1 ) observed in the ' $1 / 1$ ' approximant, a phase that is stable over a range of Cd-rich systems [6], a natural approach to modelling the quasicrystal structure is to generalize the cluster packing rule seen in the $1 / 1$ approximant to icosahedral symmetry. Intercluster linkages run along twofold and

*Corresponding author. Email: widom@andrew.cmu.edu 

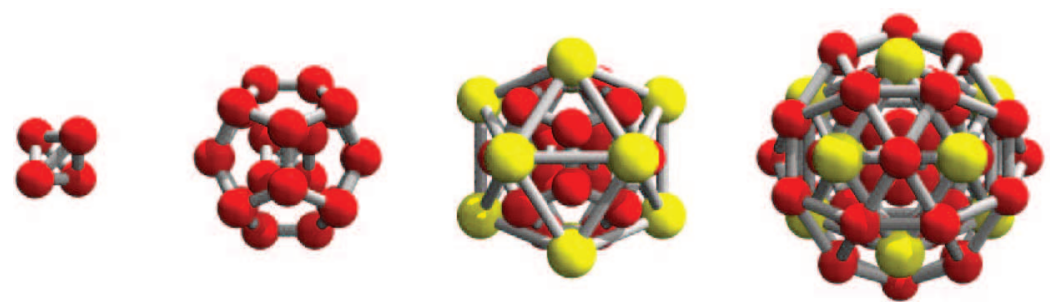

Figure 1. Adding shells to a $\mathrm{Ca}_{12} \mathrm{Cd}_{54}$ cluster. Left to right: (s1) $\mathrm{Cd}_{4}$ tetrahedron; (s2) $\mathrm{Cd}_{20}$ dodecahedron; (s3) $\mathrm{Ca}_{12}$ icosahedron; (s4) $\mathrm{Cd}_{30}$ icosidodecahedron.

threefold directions. A set of space-filling and non-overlapping cells based on these linkages, admitting long-range icosahedral order, is canonical cell tiling (CCT) [7]. We employ small approximants of this tiling to study all plausible ways of packing the clusters systematically, while refining simultaneously structural model using $a b$ initio total energy calculations. Use of a CCT tiling model is further justified by the fact that the '2/1' $\mathrm{Ca}-\mathrm{Cd}$ approximant [8] turns out to be a CCT approximant also. An analogous ab initio heat-energy structure refinement has been carried out for a Frank-Kasper-type Ti-Cr-Si quasicrystal [9], also utilizing a CCT decoration approach.

$\mathrm{Cd}$ based quasicrystals create interest both because of their unusual structure and because they occur as binary alloys. Binary alloy systems can be simpler to study, both experimentally and theoretically, than the ternary alloy systems in which previously known thermodynamically stable quasicrystals occur. One advantage of a binary alloy is the presence of just a single composition variable to control, as opposed to two independent compositions in a ternary. Another advantage, from a theoretical perspective, is the strong size constrast between the large $\mathrm{Ca}$ or $\mathrm{Yb}$ atoms (nominal diameters, 3.95 and $3.88 \AA$ ) and the relatively small $\mathrm{Cd}$ atoms (nominal diameter, 2.98 $\AA$ ). These elements also exhibit strong differences in electronegativity and other chemical properties. As a result, chemical substitution resulting in mixed site occupancy seems unlikely. In contrast, at least one pair of elements substitute easily for each other in the stable ternary quasicrystals.

\section{Canonical cell approximants and competing $\mathrm{Ca}-\mathrm{Cd}$ phases}

Our $\mathrm{Ca}-\mathrm{Cd}$ decoration models start from the assumption that the basic clusters found in the two known approximant compounds $\mathrm{CaCd}_{6}$ and $\mathrm{Ca}_{13} \mathrm{Cd}_{76}$ occur with maximal frequency also in the icosahedral phase, and with the same packing rule observed in the two aforementioned structures. The packing rule is that clusters link along twofold and threefold icosahedral directions, thus maintaining long-range order. The same scenario motivated formulation of the CCT [7]. Space is divided into four kinds of cell, denoted A, B, C, and D (figure 2). Cell vertices are occupied by icosahedral clusters linked along twofold and threefold icosahedral directions, which are called $b$ and $c$ bonds respectively. The A cell is a bcc tetrahedron, the $\mathrm{B}$ cell is a rectangular pyramid, the $\mathrm{C}$ cell is a triangular tetrahedron and the $\mathrm{D}$ cell is a trigonal prism. Cell faces are of three types: $\mathrm{X}$ (isosceles triangle with one $b$ and 

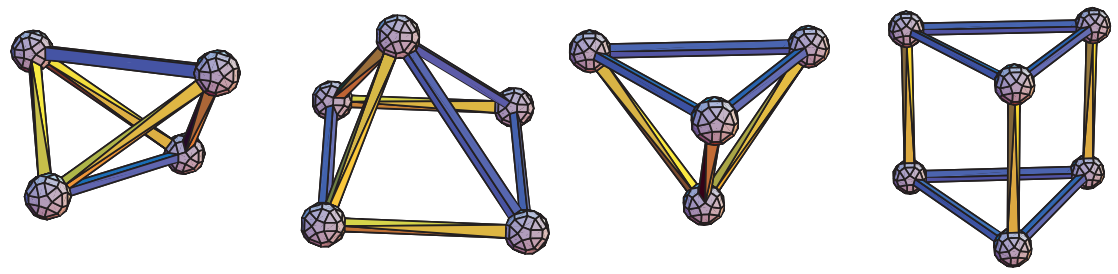

Figure 2. Canonical cells [7] (left to right, A, B, C and D) illustrated as if constructed from ZomeTools. Icosahedral balls occupy vertices. Blue and yellow edges lie along twofold and threefold directions respectively.

Table 1. CCTs considered in this work: the Pearson symbol of the tiling, the names of known compound, the space groups, the numbers of objects per unit cell, the first-principles energies (GGA, generalized gradient approximation; LDA, local-density approximation), total number $N_{\text {tot }}$ of atoms.

\begin{tabular}{|c|c|c|c|c|c|c|c|c|c|c|}
\hline $\begin{array}{l}\text { Pearson } \\
\text { (symbol) }\end{array}$ & Compound & $\begin{array}{l}\text { Space } \\
\text { group }\end{array}$ & Nodes & A & B & $\mathrm{C}$ & $\mathrm{D}$ & $\Delta E(\mathrm{GGA})$ & $\Delta E(\mathrm{LDA})$ & $N_{\text {tot }}$ \\
\hline A.cI2 & $\mathrm{CaCd}_{6}$ & $\operatorname{Im} \overline{3}$ & 2 & 12 & 0 & 0 & 0 & Stable & Stable & 168 \\
\hline BC.hR1 & & $R \overline{3} m$ & 1 & 0 & 2 & 2 & 0 & 5.5 & 3.3 & 94 \\
\hline D.hP1 $1_{\mathrm{Cd}}$ & & $P \overline{3} m$ & 1 & 0 & 0 & 0 & 1 & 8.8 & 5.5 & 126 \\
\hline D. $h P 1_{\mathrm{Ca}}$ & & $P \overline{3} m$ & 1 & 0 & 0 & 0 & 1 & 12.6 & 10.5 & 126 \\
\hline ABC.cP8 & $\mathrm{Ca}_{13} \mathrm{Cd}_{76}$ & $P a \overline{3}$ & 8 & 24 & 8 & 8 & 0 & 4.4 & & 712 \\
\hline ABC.mP2 & & $P 2$ & 2 & 6 & 2 & 2 & 0 & 8.0 & & 178 \\
\hline ABC.mP4 & & $P 2_{1} / c$ & 4 & 12 & 4 & 4 & 0 & 8.1 & & 356 \\
\hline ABCD.hR3 & & $R 3 m$ & 3 & 6 & 3 & 3 & 1 & 4.1 & & 288 \\
\hline ABCD.mP4 & & $P 2 / m$ & 4 & 6 & 4 & 4 & 2 & 8.3 & & 398 \\
\hline $1 / 1 \mathrm{Al}-\mathrm{Cu}-\mathrm{Li}$ & & $\operatorname{Im} \overline{3}$ & 2 & 12 & 0 & 0 & 0 & & 0.9 & 160 \\
\hline
\end{tabular}

two $c$ edges); Y (equilateral triangle with three $b$ edges); $\mathrm{Z}$ (rectangle with two $b$ and two $c$ edges). Packing constraints guarantee that B and $C$ cells occur in equal numbers. A special degree of freedom of the icosahedral CCT is that a fraction of A cells may be converted into D cells (or vice versa).

In the following we consider the energetics of several CCTs (table 1) together with competing $\mathrm{Ca}-\mathrm{Cd}$ phases. All model structures considered in table 1 and figure 3, and their energies, are available from [10].

Two of the tilings correspond to known $\mathrm{Ca}-\mathrm{Cd}$ structures. The $\mathrm{CaCd}_{6}$ structure, also known as the $1 / 1$ cubic approximant, is the pure A-cell tiling A.cI2. The precise structures remain controversial with the Pearson symbol given variously as cI160, cI176, cI184 and cI232 [4, 6, 11-14] as a result of uncertainty regarding the proper orientations of the $\mathrm{Cd}_{4}$ cluster centre tetrahedra. In fact, there appears to be a low-temperature (around $T=100 \mathrm{~K}$ ) order-disorder phase transition [14-16] in which these tetrahedra lock into specific relative orientations. Calculations $[17,18]$ confirm that ordering into particular cluster orientations can lower the energy slightly but that at elevated temperatures this degree of freedom should indeed be disordered; so the structure bcc, with two basic $\mathrm{Ca}_{12} \mathrm{Cd}_{54}$ clusters plus $36 \mathrm{Cd}$ glue atoms per sc cell. 


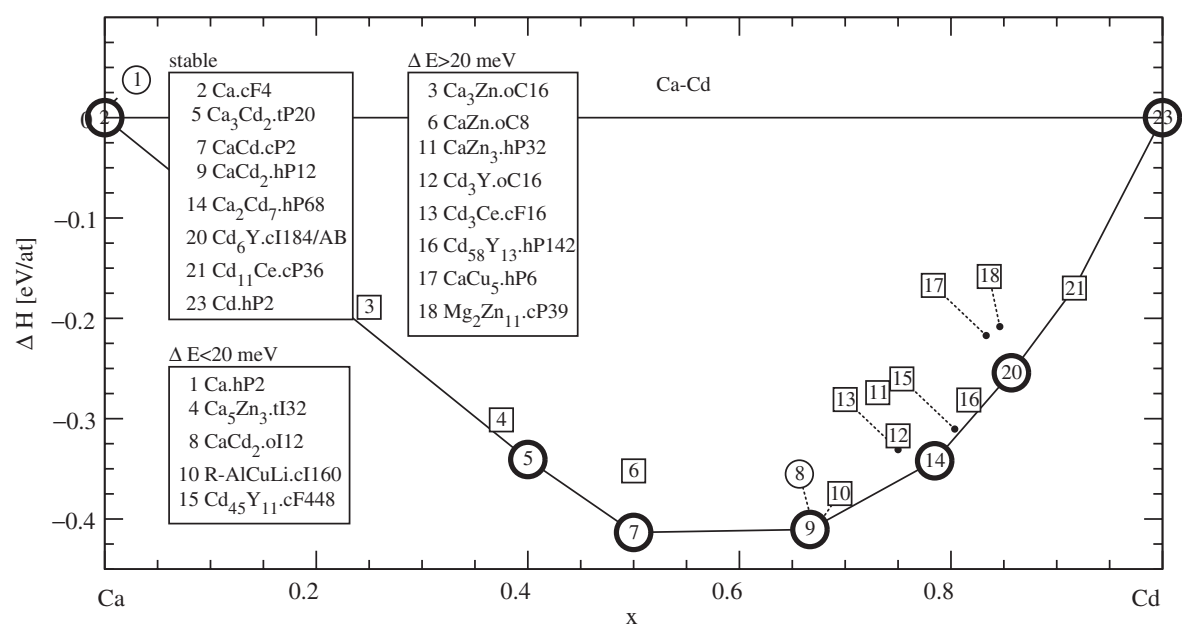

Figure 3. Enthalpies of formation of $\mathrm{Ca}-\mathrm{Cd}$ compounds. Thick open circles indicate known low-temperature phases; thin open circles indicate known high-temperature phases; open squares indicate either structures not reported in the $\mathrm{Ca}-\mathrm{Cd}$ system. Line segments connect the vertices of the convex hull. The keys list the calculated stable structures $(\Delta E \equiv 0)$, low- but positive-energy structures $(0<\Delta E<20)$ and higher-energy structures $(20<\Delta E)$. Additional data and structure details are available in [10].
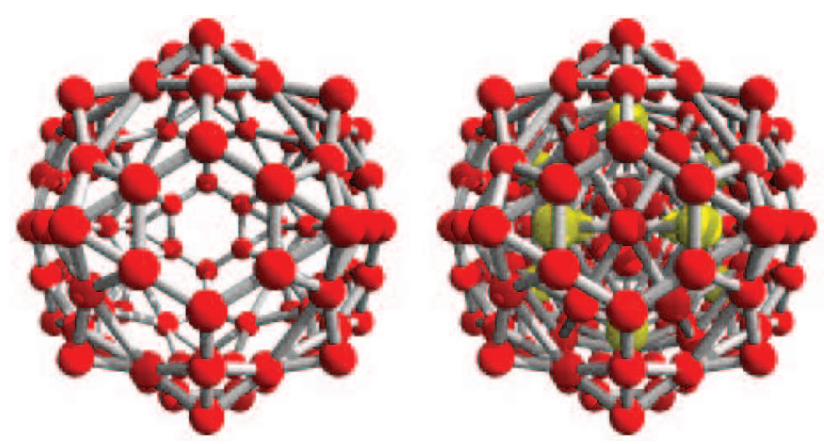

Figure 4. Outer shell (Ss) of the cluster.

The bcc structure tiles space with canonical A cells, with a cluster centre at each A-cell vertex. Each cubic cell contains 12 A cells. The 1/1 approximant structure implies a unique decoration of the A cell, save for the cluster center tetrahedra. The other solved approximant structure [8] is the $2 / 1$ cubic approximant, $\mathrm{Ca}_{13} \mathrm{Cd}_{76}$. We recognize this as the ABC.cP8 tiling, containing $24 \mathrm{~A}$, eight $\mathrm{B}$ and eight $\mathrm{C}$ cells. The decoration implied for the A cell agrees precisely with that obtained from the $1 / 1$ approximant. A new feature of the structure is the double Friauf polyhedron [8] occurring around pairs of $\mathrm{Ca}$ atoms that are not part of the s3 Ca shell and occur near the centres of Y faces. A more detailed description is given in section 4.

In the $1 / 1$ approximant, $\mathrm{Ca}$ atoms are never in contact with each other. In the $2 / 1$ approximant, with slightly higher $\mathrm{Ca}$ content, the new $\mathrm{Ca}$ environment is a double 
Friauf polyhedron, with one $\mathrm{Ca}-\mathrm{Ca}$ contact. Since the icosahedral phase occurs at even slightly higher $\mathrm{Ca}$ contents, it is interesting to inspect the structures of another two $\mathrm{CaCd}$ phases with higher $\mathrm{Ca}$ contact. The $\mathrm{Ca}_{2} \mathrm{Cd}_{7} \cdot \mathrm{hP} 68$ structure has $14 \mathrm{Ca}$ atoms on three Wyckoff sites; two of these (12 $\mathrm{Ca}$ at $\mathrm{Ca}(5)$ and $\mathrm{Ca}(7))$ have no $\mathrm{Ca}-\mathrm{Ca}$ contacts, while the $\mathrm{Ca}(2)$ site (two atoms) is the same double Friauf polyhedron found in the $2 / 1$ approximant. The $\mathrm{CaCd}_{2} \cdot \mathrm{hP} 12$ Laves phase has a single type of $\mathrm{Ca}$ environment, a Friauf polyhedron with four $\mathrm{Ca}-\mathrm{Ca}$ contacts per $\mathrm{Ca}$ atom.

\section{Total energy calculations}

We carry out ab initio calculations using the plane-wave program Vienna Ab initio Simulation Package (VASP) [19, 20] which yields reasonably accurate total energies. This approach uses ultrasoft pseudopotentials [21, 22] and projector augmentedwave (PAW) potentials [23] to represent the effective interaction of valence electrons with ionic cores and solves the many-body quantum-mechanical band structure of these electrons using electronic density functional theory. We choose to model $\mathrm{Cd}-\mathrm{Ca}$ rather than $\mathrm{Cd}-\mathrm{Yb}$ because the alkaline earth element $\mathrm{Ca}$ is easier to treat from first principles than the rare-earth element $\mathrm{Yb}$.

First, we use VASP to reproduce the sequence of low-temperature stable phases in the established $\mathrm{Ca}-\mathrm{Cd}$ binary phase diagram [24]. To do this we calculate the cohesive energy for each known structure, and several hypothetical strctures. Each structure is fully relaxed in both unit-cell parameters and atomic coordinates. All energies are converged to an accuracy of $1 \mathrm{meV}$ atom $^{-1}$ or better by increasing the $k$-point mesh density. These calculations use PAW potentials in both the local-density approximation (LDA) and the generalized gradient approximation (GGA). All calculations are performed with a constant plane-wave energy cut-off of $274 \mathrm{eV}$.

For the largest of our approximant structures (ABC.cP8 tiling; 712 atoms in cubic cell), the relaxations presented a formidable computational task. The $\Gamma$-point-only calculation required about 16 Gbytes memory, and one ionic step took a central processing unit time of about $12-20 \mathrm{~h}$ on eight Opteron processors. The final energy reported in table 1 refers to a structure pre-relaxed using embedded-atom method (EAM) potentials [25], and final VASP calculation at $K=(1 / 4,1 / 4,1 / 4)$ to avoid any spurious effect arising from the highly symmetric $\Gamma$-point calculation.

Subtracting each cohesive energy from the tie line joining the pure elements in their ground states yields enthalpies of formation, $\Delta H_{\text {for }}($ at $T=0 \mathrm{~K}$ ). Enthalpies of all known and many hypothetical $\mathrm{Ca}-\mathrm{Cd}$ structures are plotted in figure 3 . We label each structure with its name followed by its Pearson symbol.

Agreement between our calculation and the established phase diagram requires that all known low-temperature structures lie on the convex hull of enthalpy versus compositional parameter. Additionally, all hypothetical structures must lie above the convex hull (we define $\Delta E$ as a measure of energy above the convex hull), as must all known high-temperature, high-pressure and metastable phases.

Agreement is good but not perfect, because we find that $\mathrm{Cd}_{11} \mathrm{Ce} . \mathrm{PP} 36$ touches the convex hull, predicting that it should occur as a stable phase. This structure has 
not been reported in $\mathrm{Ca}-\mathrm{Cd}$. We find it to be marginally stable, lying $4.6 \mathrm{meV}$ atom $^{-1}$ below the line from $\mathrm{CaCd}_{6}$ to pure $\mathrm{Cd}$; so small errors in our calculations could cause us to mispredict stability. Alternatively, it may be truly stable but difficult to nucleate and grow.

We also encounter substantial trouble with $\mathrm{CaCd}_{2}$, when we use the GGA. Experimentally the $\alpha-\mathrm{CaCd}_{2} \cdot \mathrm{hP} 12$ phase is stable at low temperature, while the $\beta-\mathrm{CaCd}_{2}$.oI12 phase is stable at high temperatures. However, using the GGA we find oI12 lower in energy than hP12 by $12 \mathrm{meV}$ atom $^{-1}$, incorrectly predicting that the $\beta$ phase should be stable at low temperatures. In contrast, we find oI12 higher in energy than hP12 by $15 \mathrm{meV}$ atom $^{-1}$ using LDA, consistent with experimental observation. We have not seen this level of disagreement between LDA and GGA in any other alloy system studied to date. Additionally, the GGA should in principle be more accurate than the LDA, but in the present case it seems to be less accurate. Possibly a relativistic treatment of $\mathrm{Cd}$ would be helpful [18]. Fortunately, $\mathrm{CaCd}_{2}$ is the only case where a qualitative difference emerges between LDA and GGA. It seems that our methods yield sufficient accuracy when applied to the CCT approximants, although we continue to see a significant tendency for GGA to disfavour $\mathrm{Ca}-\mathrm{Ca}$ contacts more strongly than the LDA.

\section{Canonical cell tiling decoration refinement and new structures}

Given a specific tiling, modelling the icosahedral $\mathrm{Ca}-\mathrm{Cd}$ atomic structure naturally breaks into two parts:

(i) refining the decoration outside the clusters;

(ii) determining the optimal cluster center tetrahedron orientations.

First, we describe step (1).

Technically, our model was implemented as a decoration rule for CCT 'objects' (vertices, bonds, faces and cells) following the method described in [26]. The positional parameters of a decoration 'orbit' are constrained by point symmetry of tiling object associated with it. We start by registering atomic sites given by known $1 / 1$ and $2 / 1$ structures with tiling objects. Because of the complexity of the decoration rule that partially captures displacive symmetry breakings invoked by cluster tetrahedral cores, in this paper we give only a simple approximate 'topological' decription, which covers all atoms, some of them more than once. This description is based on extending the fundamental cluster by another shell, s5, as proposed in [5] and illustrated in figure 4 . This shell exists as a complete, although overlapping unit in the 1/1 approximant. In a general CCT (including the $2 / 1$ approximant), only a subset of these sites are actually occupied by atoms.

The shell s5 may be geometrically represented by a rhombic triacontahedron (RT) of radius about $8 \AA$. It is decorated by Cd atoms on its 12 fivefold and 20 threefold vertices, as well as 60 mid edge points, yielding-total of 92 atoms. The RT decorating CCT vertices share oblate rhombohedra along each threefold $c$ linkage, as well as rhombic faces along twofold $b$ linkages. In [26], this structural description has been called 'dual', as the RT vertices coincide with points projected from the body centres of the six-dimensional hypercubic lattice. 
All remaining uncovered space in an arbitrary CCT is tiled by prolate rhombohedra (PRs): one PR per each Z face, and one PR per each D cell. Thus, the $1 / 1$ approximant structure is covered completely by overlapping RT, while the $2 / 1$ approximant (ABC.cP8 tiling) is covered by eight RTs plus four isolated PRs sharing all their faces with the RT.

The PRs mostly share faces with RTs; hence a natural extension of the RT decoration for PR is the familiar Frank-Kasper decoration, with atoms decorating vertices and midedge points of rhombus faces. In the Frank-Kasper quasicrystal structures [27], a large atom is always placed below and above the midpoint of the rhombic faces. In the present $\mathrm{Ca}-\mathrm{Cd}$ case, such a position is occupied by $\mathrm{Cd}$ from the inner side of the RT and is naturally complemented by the very large $\mathrm{Ca}$ atom from the PR inner sides, where two Ca atoms fall exactly on the long body diagonal. In other words, the PRs outside the RTs are decorated by interpenetrating Friauf polyhedra, as in the 2/1 approximant [8], or in CaCd2.hP12 Laves phase.

A new situation occurs in a general approximant containing D cells, namely a PR piercing a $\mathrm{Z}$ face shares three faces with three other PRs in the D-cell interior. In this situation, one $\mathrm{Ca}$ atom in the $\mathrm{PR}(\mathrm{D})$ contacts four other $\mathrm{Ca}$ atoms, as in $\mathrm{CaCd}$ 2.hP12. On the other hand, the Ca2Cd7.hP68 structure avoids multiple $\mathrm{Ca}-\mathrm{Ca}$ contacts; therefore we considered replacing this particular $\mathrm{Ca}$ atom with Cd. In the table 1 these variants are termed $D \cdot h P 1_{C a}$ (with Ca) or $D . h P 1_{C d}$ (with $\mathrm{Cd}$ ). Since the energy of the latter variant was lower, other models with D cells (ABCD series) in table 1 have this position occupied by a $\mathrm{Cd}$ atom.

To test further the energetics of the $\mathrm{CaCd} 2 . \mathrm{hP} 12 \mathrm{Ca}$ environments (Friauf with four $\mathrm{Ca}-\mathrm{Ca}$ contacts), we calculated the total energy of the $1 / 1$ ( $\mathrm{Al}, \mathrm{Cu}$ ) $\mathrm{Li}$ approximant $\mathrm{R}-\mathrm{Al}_{5} \mathrm{CuLi}_{3}$, substituting $\mathrm{Li} \rightarrow \mathrm{Ca}$ and $(\mathrm{Al}, \mathrm{Cu}) \rightarrow \mathrm{Cd}$ (last entry in the table). In this stucture, the Friauf polyhedra are composed of Bergman clusters with empty centres, packed on the standard bcc lattice. With $32.5 \%$ of $\mathrm{Ca}$ atoms, the structure competes with $\mathrm{CaCd} 2 . \mathrm{hP} 12$, being slightly unstable by about $1 \mathrm{meV}$ atom ${ }^{-1}$ according to the LDA.

Initial tetrahedron orientations in our models were decided by hand for the $1 / 1$ approximant, and by fixed-site Monte Carlo annealing, using a toy pair potentials in other cases. The 'site list' of candidate $\mathrm{Cd}$ positions was derived from experimental observations [6]. For the larger approximants, we performed a low-temperature molecular dynamics annealing using EAM potentials [25], prior to full $a b$ initio relaxation. Interestingly, during the low-temperature molecular dynamics, Cd atoms from the cluster centre tetrahedra can traverse from one cluster to another by pushing s2 atoms out of their nominal positions into 'cube-center' positions [6] located on the threefold linkage between neighbouring clusters. These sites may serve as a low-energy transient position for a $\mathrm{Cd}$, and indeed such sites have non-zero occupancy in the 2/1 approximant.

$\Delta E$ values of our optimal decorations, listed for the tilings in table 1, vary from +3 to $+9 \mathrm{meV}^{-1}$ atom $^{-1}$. We attribute most of the energy variation to the problem of cluster centre tetrahedron orientations. However, in the case of larger approximants, incomplete $k$-point convergence and structural relaxation may also contribute. In the cases of very large structures, we carried out some pre-relaxation using EAM potentials [25], because the ab initio calculations were time and memory consuming. 


\section{Discussion}

We choose to model the $\mathrm{Ca}-\mathrm{Cd}$ quasicrystal structure using real three-dimensional space rather than the six-dimensional space that is commonly used for quasiperiodic icosahedral structures. One reason for doing so is that many sites cannot be conveniently represented in the six-dimensional space; so the atomic surface structure would have to be quite complicated. Additionally, our calculation of energetics demands a focus on real-space structure, especially because the smaller approximants that are in reach of first-principles calculation do not sample perpendicular space extensively. Yet, they provide all the variety needed to resolve tile decoration rules in real space.

Our final model yields a deterministic assignment of glue atoms within canonical cells. The cluster centre tetrahedra are not deterministic, and follow contextdependent rules as yet undiscovered. We have checked that the EAM Ca-Cd potentials describe accurately $\mathrm{Ca}-\mathrm{Cd}$ energetics around the composition of interest. Resolving interesting questions regarding ordering of the tetrahedra inside the cluster motifs requires study of supercell models with large numbers of atoms and appears to be beyond reach within the $a b$ initio calculational approach. However, this task may be possible using the EAM potentials.

The energetics of large-atom contacts (e.g. Ca-Ca) may be crucial for quasicrystal formation. Consider the phase diagrams of several $\mathrm{Cd}-\mathrm{M}$ compounds $(\mathrm{M}=$ large atom, specifically Ca, Yb, Ce, Gd, Sm, Eu, Y, Ba, Nd, Pr, Dy, Er or Sc). Stable iQCs form in compounds in which hP68 is the stable structure around the composition at parameter $x_{\mathrm{Cd}}=0.80$ (e.g. $\mathrm{Cd}-\mathrm{Ca}$ and $\mathrm{Cd}-\mathrm{Yb}$ ), rather than $\mathrm{hP} 142$ or $\mathrm{cF} 448$. The latter two structures avoid M-M contacts, while one of the seven M atoms in hP68 occurs in double Friauf polyhedra with one $\mathrm{M}-\mathrm{M}$ contact per atom. At the same time, at the composition $\mathrm{Cd}_{2} \mathrm{M}$, iQC-forming binaries $\mathrm{Ca}-\mathrm{Cd}$ and $\mathrm{Cd}-\mathrm{Yb}$ form the hP12 Laves phase in which each $\mathrm{M}$ atom occur in Friauf-polyhedron-like local environments and participates in four $\mathrm{M}-\mathrm{M}$ contacts. Meanwhile, other $\mathrm{Cd}-\mathrm{M}$ compounds form hP3 structures with only two M-M contacts or oI12 with two $\mathrm{M}-\mathrm{M}$ contacts and two other near-contacts. We speculate that the stability of $\mathrm{M}-\mathrm{M}$ contacts within Friauf polyhedra may tip the balance towards the formation of the quasicrystal phase.

If it is indeed true that energetics of $\mathrm{M}-\mathrm{M}$ contacts influences the stability of the iQC phase, then it is unfortunate that our VASP GGA calculations reproduce their energy poorly. Further study is needed to clarify the inconsistencies at the $\mathrm{Cd}_{2} \mathrm{Ca}$ composition. A pseudopotential-free method that incorporates relativistic effects, [18] may be more reliable. Experimental tests for $\mathrm{M}-\mathrm{M}$ contacts in iQCs would be valuble, as would diffraction studies of $\mathrm{M}-\mathrm{M}$ correlations in the molten liquid alloy [28].

\section{Acknowledgements}

This work was supported in part by National Science Foundation grant DMR0111198. M. M. also acknowledges support from grants no. VEGA-2/5096/25, APVT-51021102, APVT-51052702 and SO-51/03R80603. Ab initio calculations 
were carried out under EC-funded project HPC-Europa, contract 506079, and at the Pittsburgh Supercomputer Center. We thank Veit Elser for his IQtools program used to create figure 2, we thank P. Brommer and F. Gähler for use of their EAM potentials and we thank C.P. Gomez, H. Takakura and E. Zijlstra for useful discussions.

\section{References}

[1] A.P. Tsai, J.Q. Guo, E. Abe, et al., Nature 408537 (2000).

[2] J.Q. Guo, E. Abe and A.P. Tsai, Phys. Rev. B 62 R14 605 (2000).

[3] J.Z. Jiang, C.H. Jensen, A.R. Rasmussen, et al., Appl. Phys. Lett. 781856 (2001).

[4] H. Takakura, J. Guo and A.P. Tsai, Phil. Mag. Lett. 81411 (2001).

[5] H. Takakura, C. Gomez, A. Yamamoto, et al., Phil. Mag. 86621 (2006).

[6] C.P. Gomez and S. Lidin, Phys. Rev. B 68024203 (2003).

[7] C.L. Henley, Phys. Rev. B 43993 (1991).

[8] C.P. Gomez and S. Lidin, Angew Chem., Int. Ed. 404037 (2001).

[9] R.G. Hennig, A.E. Carlsson, K.F. Kelton, et al., Phys. Rev. B 71144103 (2005).

[10] Alloy database. Available online at: http://alloy.phys.cmu.edu (accessed 2005).

[11] A. Palenzona, J. Less-Common Metals 25367 (1971).

[12] G. Bruzzone, Gazz. Chim. Ital. 102234 (1972).

[13] Y. Ishii and T. Fujiwara, Phys. Rev. Lett. 87206408 (2001).

[14] R. Tamura, K. Edagawa, Y. Murao, et al., J. Non-Crystalline Solids 334-335 173 (2004).

[15] R. Tamura, Y. Murao, S. Takeuchi, et al., Jap. J. Appl. Phys. 41 L524 (2002).

[16] R. Tamura, K. Edagawa, C. Aoki, et al., Phys. Rev. B 68174105 (2003).

[17] M. Widom and M. Mihalkovič, Mater. Res. Soc. Symp. Proc. 805 LL 1.10 (2004).

[18] E.S. Zijlstra, S.K. Bose and R. Tamura, Phys. Rev. B 70184205 (2004).

[19] G. Kresse and J. Hafner, Phys. Rev. B 47 RC558 (1993).

[20] G. Kresse and J. Furthmuller, Phys. Rev. B 5411169 (1996).

[21] D. Vanderbilt, Phys. Rev. B 417892 (1990).

[22] G. Kresse and J. Hafner, J. Phys.: Condens. Matter 68245 (1994).

[23] G. Kresse and J. Joubert, Phys. Rev. B 591758 (1999).

[24] T.B. Massalski (Editor), Binary Alloy Phase Diagrams (ASM International, Materials Park, Ohio, 1990).

[25] P. Brommer and F. Gāhler, Phil. Mag. 86753 (2006).

[26] M. Mihalkovič, W.J. Zhu, C.L. Henley, et al., Phys. Rev. B 539002 (1996).

[27] C.L. Henley and V. Elser, Phil. Mag. B 53 L59 (1986).

[28] M.J. Kramer, T.A. Lograsso and D.J. Sordelet, Phil. Mag. Lett. 85151 (2005). 